\title{
Networked Simulators and Computer-Supported Wargame Simulations
}

\author{
Thomas W. Mastaglio \\ U.S. Army Training and Doctrine Command \\ Fort Monroe, VA 23651
}

\begin{abstract}
There is misunderstanding in both the training and research communities of how the two major technological approaches to simulated training environments that support military collective training of units differ. This paper compares the networking of simulators approach to computer-supported wargaming first in a cognitive perspective. Then they are compared in terms of the computer technologies they use, both in their hardware and software designs. I also discuss some significant directions for basic research to improve both approaches.
\end{abstract}

\section{INTRODUCTION}

The development of networked supported simulation technology provides the military community a new type of training environment. The Defense Advanced Research Projects Agency (DARPA) developed the simulation networking capability for the United States Army as a proof of principle technology demonstration $[1,2]$. That effort did more than achieve a successful technology demonstration, it also led to training effectiveness evaluations and assessments of the benefits offered by experiential training that takes place in a virtual environment $[3,4,5]$. We now better understand what is required to present training audiences a graphically generated representation of reality with sufficient fidelity to make training effective. More specifically, we can prescribe the technical requirements of networked simulations (also known as distributed interactive simulations) which are surrogate environments designed to replace some portions of our training on field exercises.

On the other hand, computer simulations of battle have been used to drive wargame exercises for analysis and training for almost a decade (e.g., [6]). Computer-supported wargames evolved from manual board games. Unfortunately, the availability of two similar technologies, networked simulators and computer-supported wargames, has led to confusion in the ranks of both developers and trainers. Each type of environment has unique and complementary capabilities, but the proponents of one approach or the other have too often taken the view that these are equivalent technologies. In this paper attempts to clarify the role of the two technologies in order to facilitate better understanding and

Manuscript received July 29, 1991. The contents of this paper are solely the responsibility of the author and do not represent an official position of the U.S. Army. to aid further development of each using the most promising scientific ideas. I will compare the two technologies in terms of their roles in the cognitive domain and area of computational technology.

Before laying out this comparison it is worth noting those aspects not addressed. This paper does defend or champion one approach over the other, nor make a claim that one is better. Each technology, in both its present form and more particularly with future technological advancements that get discussed, provides support for different training requirements. It is the failure to understand this on the part of supporters of both approaches that has created confusion for the training community that use and develop these systems. Neither will I discuss the integration of the two approaches, an effort that is important in its own right and of considerable interest to both the research and user communities. Also, single, stand alone simulators (i.e., an aircraft flight simulator) are not discussed here. Such simulators are used to train individual operators or single crews in operational and emergency equipment procedures. They use precanned scenarios focused on rehearsing or practising particular skills or sets of skills while both networks of simulators and computer supported wargames offer free play virtual battlefields.

\section{COGNITIVE COMPARISON}

Given hindsight, it is now apparent that beginning from a cognitive perspective, focused on the needs of the training audience and the skills to be developed, we could lay out a some fundamental goals which could provide a basis for both technology development and system design. Unfortunately most training systems are developed as applications of promising technology rather than the development orienting on meeting established requirements. In the cognitive area I will first compare distributed networked simulators with wargaming systems in terms of the training that take place, then discuss how modelling cognition in machines can be applied to enhance both computational approaches.

\section{A. LEVELS AND TYPES OF SKILLS COMPARISON}

Networked simulators, like SIMNET $[1,2]$, are ideal environments for training team skills, teams from crew through company size formations learn to use weapons systems as part of an integrated effort. Figure 1 locates the 


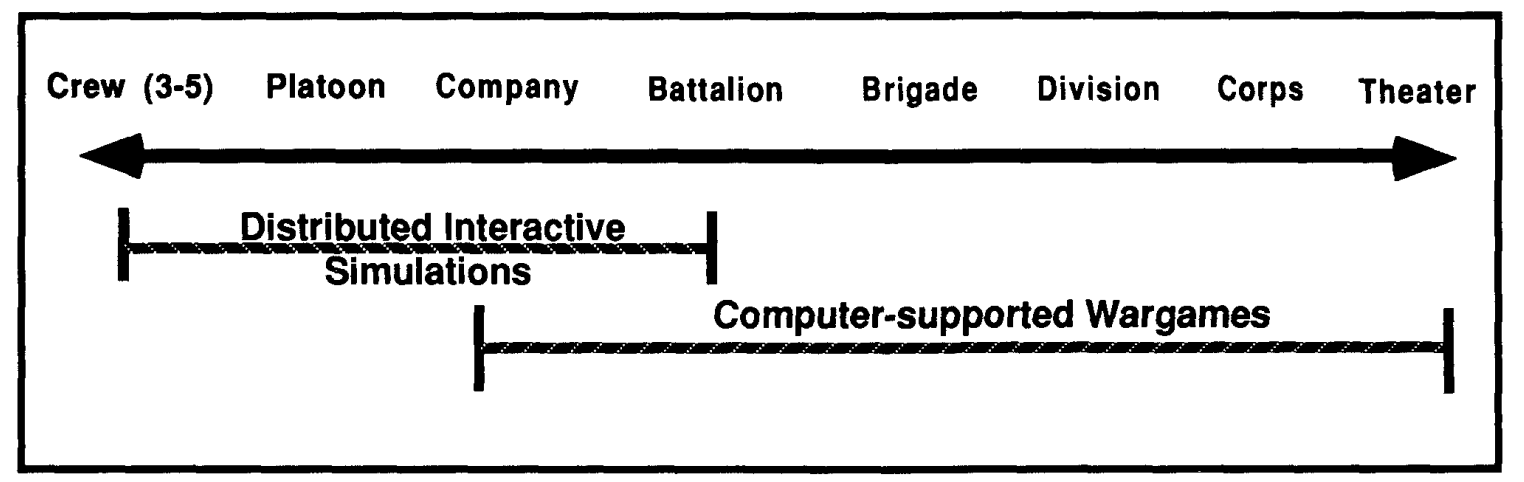

FIGURE 1.

two technologies along a continuum dimensioned by the size of the target audience. While there is overlap in the training audiences served, networks of surrogate combat systems are best suited to training procedural skills in operating that equipment in the context of a coordinated organizational effort. In distributed interactive simulation an organization is exposed to the simulated pressure of battle. The trainees rehearse their unit standing operating procedures (SOPs) under conditions designed to replicate the stress of actual combat. Also significant is that team members learn to work together as a group; they learn each others individual and the team's collective idiosyncrasies. This is an extremely important capability often overlooked when the benefits (and drawbacks) of simulations-based training are discussed. Short of warfare itself, our military organization have, in the past, had to rely on learning how to deal with and operate in their individual units through other peacetime activities (i.e., inspections, reviews, etc) or during costly field training exercises using expensive equipment. The latter are too often controlled, for safety reasons, to the point where insufficient cognitive loads are placed on the training audience.

Computer-supported wargames teach unit commanders and their staffs (in organizations which are large enough to have a staff) how to allocate and manage, in real time, the combat resources available to them. They focus on providing a realistic assessment of those decisions, both ones made during planning and those that result from the situated actions [7], that takes place once the battle begins. That assessment is done using computer models based on historical data and accepted analytical formulae [8]. The skills exercised are leadership and decision-making. This opportunity to practise staff planning, advising and the leadership decision-making process is seldom provided with equivalent seriousness in situations with real time constraints in our garrison activities. Without wargame-based exercises these conditions can only be achieved by putting progressively larger formations of subordinate units "in the field". This is expensive in terms of operational costs, environmental impact and potential for wasting the time of subordinate formations while they serve as training aids for the higher unit staff and commander.

\section{B.ROLE OF MACHINE COGNITION}

Both simulation approaches are evolving technologies that can be improved with better theoretical understanding of human cognition, and the ability to achieve cognitive capabilities in software architectures. As our understanding of human processing improves we will better understand these issues in terms of the skills which are trainable and how. Additionally, the state of research in machine cognition is such that better training can be achieved using techniques from artificial intelligence research.

After developing the capability to network simulators at the hardware and inter-processor communications levels we learned that to achieve sufficient fidelity to make it effective required software models for additional entities. These entities are software surrogates for virtual simulators, or enemy vehicles and units against which the training audience operates. Artificial intelligence techniques are used to model these entities but because it was difficult to achieve acceptable fidelity, they are called semi-automated forces, rather than intelligent agents [9]. Expanding this approach to software modelling of virtual forces is an open research agenda item. It will require the application of available approaches for modelling cognition and behavior and the development of new approaches, such as in [10] and [11]. The goal for this research is improved approaches to modelling human behavior, models which are executable in real time to drive the simulated actions of surrogate battle participants.

Besides using artificial intelligence modelling approaches, cognitive science can help improve distributed interactive simulation technology by developing improved interfaces to the virtual battlefield. The general technical approach is to provide the training audience a view port into that battlefield using display technology driven by computer image generators (CIG). Other techniques, such as virtual reality, 
which could possibly enhance fidelity and training value, must be developed and evaluated in our research programs.

An advantage to training in computer generated virtual environments over using actual equipment is the ability to digitally record the exercise, then play it back for instructor critique and student viewing. But, we have not progressed beyond simple playback capability. The application of machine intelligence to aid learning [12] is an active research area and the techniques used (modelling students, pedagogy, etc) could assist in presenting better after action reviews. An essential part of that review is the critique by the trainer. Because techniques from artificial intelligence and humancomputer interaction are needed, computer-based critiquing [13] deserves further development and integration into our systems. Other efforts to investigate better graphical aids (charts, graphs, 3-D snap shots of the battle) to support the AAR are also needed.

Technologies from research in machine cognition can also enhance training from war games supported by computers. Rule-based approaches can replace some or all of the present quantitative algorithms which are used to adjudicate individual engagements (unit versus unit) in our present systems [14] . This can make the exercise both more realistic, and we would be able to tune it to the tactics and idiosyncrasies of a specific enemy. In war gaming systems the players seldom interact directly with computers, but rather exercise controllers execute their instructions (battle commands and orders.) This is a man-power intensive process and improving the interface to the computer for exercise controllers could reduce that requirement. In a similar vein as for networked simulators, better support for after action reviews are also possible. Needs are slightly different because the battle is not completely logged for an instant replay but rather decision recorded and the battle check-pointed at given points in time for use in the after action review. During those reviews support for instruction and critiquing of the training audience actions could be supported by software tools.

\section{TECHNOLOGY COMPARISON}

There are technical differences between the two approaches to simulating battle and in the associated technological advancements that we need to improve them. The differences can be viewed in terms of the both hardware and software approaches.

\section{A. Computer Hardware Differences}

The achievement of distributed interactive simulation based on networked simulators resulted from using distributed computation across that network. These systems depend on communications protocols and network technology within local groups of simulators, and across long haul networks linking diverse, geographically separated segments of a training audience. Computer image generation (CIG) is the second significant technology required for these systems. It is only because of the recent availability of affordable CIGs with sufficient power to portray a large enough piece of terrain (approximately 3500 meter radius around each simulator) that we are able to achieve an adequate virtual battlefield. CIG machines coupled with a central processor that interfaces with the network, and tracks the battle locally at each simulator are the essential technologies used. Desirable research advances also encompass these two areas.

Higher capacity networks that will allow modelling of more battlefield entities has the potential to increase both realism, because more weapons systems can be played in the simulation, and expand the overall scope of exercises (larger aggregated units). Better computer image generation capability could increase realism for the training audience. This does not mean that costly hardware is always required, impressive results have been achieved using less expensive technologies [15]. What is needed is the investigation of how to optimally utilize what is realistically available, while identifying potential shortcomings for future developments.

The fundamental hardware technologies needed to support war games are machines with a computational capacity able to throughput large quantities of data. Our wargames are conducted faster than in real time, but we still must account for and consider many factors than can influence the battle. This means we need large data storage capacity and hardware that can compute combat calculi using many variables. These two technologies are interrelated and point out some important research requirements. Hardware with higher capacity for and rapid access to data storage is needed. The availability of large data sets requires better numerical processing capability. A solution that will address both requirements is parallel architectures with distributed concurrent processing.

A common question is why we want to expand computational capability when we already have systems that are apparently useful. The answer is that we can improve the realism of training by considering in our computer models (and in turn forcing our staffs and commanders to consider them in their planning and operations) more of the details that can influence outcome. Too often factors are assumed away because they are implicit in the combat calculus used, this leads to training audience using "gamesmanship" to "beat the system" rather than making sound tactical decisions.

\section{B. Comparisons of Software Approaches}

Just as the computer hardware central to each technolog:" differs, so to do many of the basic software approaches. The type of software most critical to each are, to some degree, dependent upon the type of hardware utilized. Research 
opportunities to improve each approach are a function of the software techniques used.

Networked simulators use object oriented, distributed computation, graphics generation and distributed redundant databases to present the virtual portion on the battlefield surrounding each simulator. An object-oriented, messagepassing paradigm models each simulator as an object on the network [16]. Each object communicates its current status to the rest of the network using scheduled broadcast procedures. This eliminates the need for central data repositories that would need continuous update. This means that we do not have to concern ourselves with redundancy, deadlock, and other issues associated with shared data. However, the approach requires every object (simulated combat system) on the network to be able to process or ignore a particular message.

Graphics generations techniques are critical to portraying the virtual battlefield. Whatever occurs within the view of any given simulator must be displayed so that crews respond accordingly. Graphics generation algorithms are triggered first by the broadcast messages received at the simulator. Status reports from other elements or nodes (friendly and enemy units/vehicles) are checked to determine which pertain to elements that are displayed in the local field of view. Secondly, information about the battle context, that is the terrain on which the operation is taking place, comes from a local terrain database. Each simulator has its own copy of that terrain data; each has a redundant copy of the terrain database. To solve the problem of consistency, present implementations do not allow changes to the terrain databases, i.e., one cannot dig a hole into which a tank can be driven. We need technical methods that overcome this restriction.

Distributed interactive systems can benefit from improved database technologies that allow both rapid access to, and update of, a central database. Such a database approach would have to be able to serve all machines without concurrency problems. An alternative is to develop techniques for simultaneous update of the databases at each node. The alternative will have to insure data consistency and prevent overloading the network. Other technological advancements that would help improve these systems include more efficient real team rendering algorithms for graphics generations and extensions to the object oriented paradigm and associated networking protocols to improve inter-simulator communications. Work is being done on standardizing protocols so that virtual battlefields can be created by networking together any number of simulators conforming to those standards [17].

Computer-assisted wargames rely on different, but not necessarily alternative, technologies. The software used to drive these exercises supports large databases with detailed information about individual units, terrain, and equipment that can influence the outcome of a battle. It implements numerical algorithms which are based on accepted combat resolution models [8]. During the progress of the battle numerous changes occur to the information in the database, such as, unit strengths, locations, available supplies and etc. These systems must keep that data current and preclude concurrent update problems. Speed of access must be sufficient to allow the model to run faster than real time.

Concurrent operations on distributed portions of such a database could allow us to more efficiently capture and use a greater quantity of data. The resolution of the wargame, i.e., the level at which we capture our units in databases, and represent them at the user-machine interface, is restricted by both data capacities and overall size of the exercise. To date, our systems can model several thousand units. This is not large enough, without aggregating into higher echelon units, to conduct exercises above an Army Corps level. It is this tradeoff between size of modelled units and size of exercise desired (or possible) that limits present training.

The battle calculus uses the information in the databases to determine the simulated results of a force-on-force engagement. The more details available about the conditions under which a battle occurs, the more realistic the results of the calculus. As database capabilities grow to replicate these larger data sets, the algorithms which apply that data to determine outcomes will increase in complexity, requiring more processing power or time. This points to another basic research problem, more efficient mathematical approaches.

Lastly the computer interface in wargaming systems is most often at the game controller level, approaches to humancomputer interaction that will make their job simpler will provide significant improvements to the training benefits derived. Current approaches are mostly command language oriented; some systems use menu-like approaches [6], and in future systems most developers intend to move to standard window-based protocols using interactive screens and menus.

\section{CONCLUSIONS}

Distributed interactive simulations and computer-supported wargaming have been show to provide effective training transfer $[18,19,20]$. Technological innovations are not needed to achieve minimum effectiveness or efficiency, but rather to improve the fidelity of these systems, fidelity at both the level of modelling of the elements involved in the battle, and in the graphics displays. Systems of the future will be comprehensive networks of simulations, networks that interconnect nodes both within one of the two discussed categories, and also between those categories. The goal of the military training community is to create large scale distributed networked simulations able to replicate complete theaters of war. To achieve such capabilities will require that we understand the cognitive requirements of our training 
audiences and can model within our machines appropriate aspects of cognition. We also want to enhance the hardware and associated software approaches to generating graphics, storing and accessing databases, providing networking communications methods and protocols, and performing large scale numerical computations.

Computer simulations are not limited to support for military training of their units, that is, collective training, but they are a fundamental technology that can be used in a variety of training environments [21]. At a more general level, claims have been made that an important role of computers in education is creating effective realistic simulations in which students experience and learn from reallife [22]. The development of simulations to support collective training in the military has pioneered the essential technologies. That effort can be studied to help us understand how simulations are best used, what cognitive principles are involved, the computer technologies they require, and the opportunities for future research to further enhance their capabilities.

\section{REFERENCES}

[1] J.A. Thorpe, "Warfighting from SIMNET," Proceedings of 10th InterservicelIndustry Training Systems Conference, 1988.

[2] J.A. Thorpe, "The New Technology of Large Scale Simulator Networking: Implications for Mastering the Art of Warfighting," Proceedings of 9 th Interservice/Industry Training Systems Conference, 1987.

[3] D.W. Bessemer, "Transfer of SIMNET Training in the Armor Officer Basic Course," U.S. Army Research Institute for the Behavioral and Social Sciences Technical Report 920, January 1991.

[4] B.L. Burnsides, "Assessing the Capabilities of Training Simulations: A Method and Simulation Networking Capability," U.S. Army Research Institute for the Behavioral and Social Sciences Research Report 1565, June 1990.

[5] E.H. Drucker, and D. A. Campshure, "An Analysis of Tank Platoon Operations and Their Simulation on Simulation Networking (SIMNET)," Ft. Knox Field Unit, Training Research Laboratory, U.S. Army Research Institute for the Behavioral and Social Sciences Research Product 90-22, 1990.

[6] R.M. Toms, L. Dobbs, and J.E. Pimper, "High-Resolution Combat Simulations to Support Training for Close Combat Light Applications," Proceedings Close Combat (Light) Simulation Technology Conference, Albuquerque, New Mexico, Sep 17-20, 1990, pages 201-226.

[7] L.A. Suchman, Plans and Situated Actions, Cambridge University Press, Cambridge, UK,1987.

[8] J.G. Taylor, Lanchester Models of Warfare, Operations Society of America, Arlington, VA, 1983.
[9] R. Brooks, B.G. Buchanan, D.B. Lenat, D.M. McKeown, JR, and J.D. Fletcher, Panel Review of the Semi-Automated Forces, Institute for Defense Analysis Document D-661 prepared for Defense Advanced Research Projects Agency, Sep 1989.

[10] K. Gates, F. Frantz, "Semi-Automated Forces Simulation Using a Blackboard," Proceedings of 12 th Interservice/Industry Training Systems Conference, November 1990, pages 295-300.

[11] M.D. Petty, R. Karr, D.R. VanBrackle, D.D. Cross, R.W. Franceschini, G.L. Gonzalez, "Functional Specification and Implemented Capabilities of the IST Semi-Automated Forces Dismounted Infantry System," Institute for Simulation and Training, University of Central Florida, Report TR 91-20, May 1991.

[12] D.H. Sleeman, J.S. Brown, "Intelligent Tutoring Systems", Academic Press, New York, Computer and People Series, 1982.

[13] G. Fischer, T. Mastaglio, "Computer-Based Critics", Proceedings of the 22nd Annual Hawaii Conference on System Sciences, Vol. III: Decision Support and Knowledge Based Systems Track, IEEE Computer Society, January 1989, pages $427-436$.

[14] Jet Propulsion Laboratory, "COBRA User's Guide," Jet Propulsion Laboratory, California Institute of Technology, Pasadena, CA, 1990.

[15] C.J. Benton, "Emerging Simulation technology: The Challenges and Capabilities of Low-Cost Technology," Proceedings Close Combat (Light) Simulation Technology Conference, Albuquerque, New Mexico, Sep 17-20, 1990, pages 90-106.

[16] T.L. Gehl, J.J. Brann, "Network Requirements for Distributed Tactical Training," Proceedings of 12 th Interservice/Industry Training Systems Conference, November 608, 1990, pages 80-91.

[17] Institute for Simulation and Training, Military Standard (Draft) Entity Information and Entity Interaction in a Distributed Interactive Simulation, Institute for Simulation and Training, University of Central Florida, Report PD 90-2, 25 January 1991.

[18] Army Research Institute for the Behavioral and Social Sciences, Issues in Psychological Research and Application of Transfer of Training, Summary of Department of Defense Roundtable organized by American Psychological Associastion, Feb 27-28, 1986.

[19] D.R. Thompson, "Transfer of Training from Simulators to Operational Equipment - Are Simulators Effective?," Journal of Educational Technology Systems, Vol 17 (3), pages 213-218, 1988-89.

[20] U.S. Army TEXCOM, Follow-On Analysis of the Close Combat Tactical Trainer (CCTT) Force Development Test and Experimentation (FDTE) Data, 1991.

[21] D.M. Towne, A. Munroe, Q.A. Pizzini, D.S. Surmon, L.E. Coller, and J.L. Wogulis, "Model-Building Tools for Simulation-Based Training," Interactive Learning Environments, Vol 1, page 33-50, 1990.

[22] R.C. Schank, M.Y. Jona, Empowering the Student, "New Perspectives on the Design of Teaching Systems," The Journal of the Learning Sciences, Vol 1, No 1, pages 7-35, 1991. 